\title{
Molecular fluorine chemistry in the early Universe
}

\author{
D. Puy ${ }^{1}$, V. Dubrovich ${ }^{1,2}$, A. Lipovka ${ }^{3}$, D. Talbi ${ }^{1}$, and P. Vonlanthen ${ }^{1}$ \\ 1 Université des Sciences Montpellier II, Groupe de Recherche en Astronomie et Astrophysique du Languedoc, GRAAL CC72, \\ 34095 Montpellier, France \\ e-mail: Denis.Puy@graal.univ-montp2.fr \\ $2 \mathrm{SPb}$ Branch of Special Astrophysical Observatory, RAS, St Petersburg, Russia \\ 3 Centro de Investigación en Física, UNISON, Rosales y Blvd. Transversal, Col. Centro, Edif. 3-I, Hermosillo, Sonora, 83000 Mexico, \\ México
}

Received 14 December 2006 / Accepted 15 August 2007

\begin{abstract}
Context. Some models of Big Bang nucleosynthesis suggest that very high baryon density regions were formed in the early Universe, and generated the production of heavy elements other than lithium such as fluorine F.

Aims. We present a comprehensive chemistry of fluorine in the post-recombination epoch.

Methods. Calculation of $\mathrm{F}, \mathrm{F}^{-}$and $\mathrm{HF}$ abundances, as a function of redshift $z$, are carried out.

Results. The main result is that the chemical conditions in the early Universe can lead to the formation of HF. The final abundance of the diatomic molecule HF is predicted to be close to $3.75 \times 10^{-17}$ when the initial abundance of neutral fluorine $\mathrm{F}$ is $10^{-15}$.

Conclusions. These results indicate that molecules of fluorine HF were already present during the dark age. This could have implications on the evolution of proto-objects and on the anisotropies of cosmic microwave background radiation. Hydride of fluorine HF may affect enhancement of the emission line intensity from the proto-objects and could produce spectral-spatial fluctuations.
\end{abstract}

Key words. cosmology: early Universe - cosmology: large-scale structure of Universe - astrochemistry

\section{Introduction}

Early Universe chemistry (standard Big Bang chemistry, hereafter SBBC), of which hydrogen recombination is only one aspect, has been the subject of several studies in the conventional frame of a homogeneous expanding Universe. One of the most important result of these studies is that the formation of a significant number of molecules such as $\mathrm{H}_{2}$ and $\mathrm{HD}$ plays a crucial role on the dynamical evolution of the first collapsing structures appearing at temperatures below a few hundred $\mathrm{K}$.

The literature on chemistry in the post-recombination epoch has expanded considerably in the recent years. Many authors have developed studies of primordial chemistry in different contexts:

- Chemical network (Lepp \& Shull 1984; Puy et al. 1993; Stancil et al. 1996; Galli \& Palla 1998; Lepp et al. 2002; Pfenniger \& Puy 2003).

- Formation of the first objects (Puy \& Signore 1996; Abel et al. 1997; Abel et al. 2000; Shchekinov \& Vasiliev 2006; Núñez-López et al. 2006; Hirata et al. 2006; Yoshida et al. 2006; Puy \& Pfenniger 2006).

All these authors clearly point out that trace amounts of light molecules such as $\mathrm{H}_{2}$, $\mathrm{HD}$ and $\mathrm{LiH}$ were formed during the postrecombination period of the Universe. However a key item is the question of heavier elements synthesis during the phase of primordial nucleosynthesis and the formation of the corresponding molecules during the period of recombination, because many observations suggest that heavy elements already exist in high redshifts (Songaila 2001; Pichon et al. 2003; Arracil et al. 2004; Cohen et al. 2004).
It is known that under certain conditions, high density baryonic bubbles are created in the Affleck-Dine model of baryogenesis (Affleck \& Dine 1985), and these bubbles may occupy a relatively small fraction of space, while the dominant part of the cosmological volume is characterized by the normal observed baryon-to-photon ratio. The value of this ratio $\eta$ in the bubbles could be much larger than the usually accepted value and still be consistent with the existing data on light element abundances and the observed angular spectrum of cosmic microwave background radiation (CMBR). In this context, numerous authors investigated the possibility that non-homogeneous baryogenesis produced very high baryon density in a small fraction of the universe and in these regions some fraction of heavy elements were already synthesized during primordial nucleosynthesis (Applegate et al. 1987; Thomas et al. 1994; Jedamzik et al. 1994; Kurki-Suonio et al. 1977; Jedamzik \& Rehm 2001). More recently Matsuura et al. (2005) analyzed Big Bang nucleosynthesis in very high baryon density regions. They found that primordial synthesis can produce very heavy elements including proton rich nuclei such as ${ }^{92} \mathrm{Mo},{ }^{94} \mathrm{Mo},{ }^{96} \mathrm{Ru}$ and ${ }^{98} \mathrm{Ru}$.

Abundance of primary fluorine is below the abundances of the primordial $\mathrm{C} \mathrm{N} \mathrm{O}$ group during the Big Bang nucleosynthesis. However molecular fluorine could be more important in comparison with the molecules provided by reactions with $\mathrm{C}, \mathrm{N}$ and $\mathrm{O}$ atoms. The first detection of interstellar hydrogen fluoride was reported by Neufeld et al. (1997) using the long wavelength spectrometer of ISO satellite. To interpret this detection of HF, they have constructed a simple model for the steady state of chemistry of interstellar fluorine with $\mathrm{H}_{2}$ and with water. Two important factors must be considered for the formation of the molecular component in the early universe. One is the binding energy of the hydrogen atom in the molecular 
component considered, and the second the ionization potential of the molecule. Relative to these two criteria, the molecule of fluorine $\mathrm{HF}$ is an interesting candidate. Ionization potential of $\mathrm{F}$ $\left(I_{\mathrm{F}}=17.42 \mathrm{eV}\right)$ is greater than that of hydrogen $\left(I_{\mathrm{H}}=13.6 \mathrm{eV}\right)$. Thus fluorine ions must have recombined before hydrogen ions in the early Universe. Moreover the binding energy of $\mathrm{HF}, E_{\mathrm{HF}}=$ $5.92 \mathrm{eV}$, is greater than the binding energy of $\mathrm{H}_{2}$. As such, $\mathrm{HF}$ could be formed in the early Universe.

In this paper we investigate the growth of molecular fluorine abundance. In Sect. 2, we introduce the standard Big Bang chemistry (hereafter SBBC). In Sect. 3, we perform a detailed analysis of the primordial chemical evolution of the atomic and molecular species of fluorine. Finally, in Sect. 5 we discuss some extensions and implications of this study.

\section{Standard Big Bang chemistry}

The standard Big Bang nucleosynthesis (SBBN) model predicts the nuclei abundances, mainly those of hydrogen, helium and lithium, and their isotopes in the early Universe. The standard chemistry of the early Universe is the chemistry of these light elements and their respective isotopic forms. This chemistry is non-trivial because on one hand the universal expansion dilutes matter, so lowers the collision rates and slows down the chemical reactions, while, on the other hand, the matter and radiation temperatures drop, which decreases the molecule destruction rates, so may encourage molecule formation.

After the hydrogen recombination, the ongoing physical reactions are numerous, partly due to the presence of the cosmic microwave background radiation (or CMBR), Stancil et al. (1998). We can establish three classes of chemical reactions:

$$
\begin{aligned}
& \text { - collisional: } \xi+\xi^{\prime} \longleftrightarrow \xi_{1}+\xi_{2} \\
& \text { - electronic: } \xi+\mathrm{e}^{-} \longleftrightarrow \xi_{1}+\xi_{2} \\
& \text { - photo-processes: } \xi+\gamma \longleftrightarrow \xi_{1}+\xi_{2} .
\end{aligned}
$$

Thus the chemical composition of the primordial gas consists of electrons and:

$$
\begin{aligned}
& \text { - hydrogen: } \mathrm{H}, \mathrm{H}^{-}, \mathrm{H}^{+}, \mathrm{H}_{2}^{+} \text {and } \mathrm{H}_{2} \\
& \text { - deuterium: } \mathrm{D}, \mathrm{D}^{+}, \mathrm{HD}, \mathrm{HD}^{+} \text {and } \mathrm{H}_{2} \mathrm{D}^{+} \\
& \text {- helium: } \mathrm{He}, \mathrm{He}^{+}, \mathrm{He}^{2+} \text { and } \mathrm{HeH}^{+} \\
& \text {- lithium: } \mathrm{Li}, \mathrm{Li}^{+}, \mathrm{Li}^{-}, \mathrm{LiH} \text { and } \mathrm{LiH}^{+} \text {. }
\end{aligned}
$$

Their respective abundances are calculated from a set of chemical reactions for the early Universe. Here we use the chemical network described by Galli \& Palla (1998). This set of ordinary differential equations depends on the radiation temperature $T_{\mathrm{r}}$ or on the matter temperature $T_{\mathrm{m}}$ and the matter density. All of these evolution equations must be solved simultaneously (Puy \& Pfenniger 2006).

The chemical kinetics of a reactant $\xi$ depends on the $\xi$ destruction, by collision with the reactant $\xi^{\prime}$ (reaction rate $k_{\xi \xi^{\prime}}$ ):

$\xi+\xi^{\prime} \rightarrow \xi_{1}+\xi_{2}$

and on the $\xi$-formation process (reaction rate $k_{\xi_{1} \xi_{2}}$ ):

$\xi_{1}+\xi_{2} \rightarrow \xi+\xi^{\prime}$

leading to the kinetic equation of the density $n_{\xi}$ :

$$
\left(\frac{\mathrm{d} n_{\xi}}{\mathrm{d} t}\right)_{\mathrm{chem}}=\sum_{\xi_{1} \xi_{2}} k_{\xi_{1} \xi_{2}} n_{\xi_{1}} n_{\xi_{2}}-\sum_{\xi^{\prime}} k_{\xi \xi^{\prime}} n_{\xi} n_{\xi^{\prime}} .
$$

In the early Universe we must also take into account the decreasing densities due to the expansion. Thus for each chemical species $\xi$ we have

$$
\frac{\mathrm{d} n_{\xi}}{\mathrm{d} t}=-3 H(t) n_{\xi}+\left(\frac{\mathrm{d} n_{\xi}}{\mathrm{d} t}\right)_{\text {chem }} .
$$

The expansion is characterized by the Hubble parameter $H(t)$, which depends on the energetic component of the Universe:

$H(t)=H_{0} \sqrt{\Omega_{\mathrm{r}}+\Omega_{\mathrm{m}}+\Omega_{\mathrm{K}}+\Omega_{\Lambda}}$

where $H_{0}=71 \mathrm{~km} \mathrm{~s}^{-1} \mathrm{Mpc}^{-1}$, given by Freedman (2000), is the present value of $H(t)$. The $\Omega_{i}$ 's density parameters depend on the redshift $z$ such as $\Omega_{\mathrm{r}} \equiv(1+z)^{4}$ (radiation density parameter) and $\Omega_{\mathrm{m}} \equiv(1+z)^{3}$ (matter density parameter). We consider here a flat Universe (i.e. $\Omega_{\mathrm{K}}=0$ ) with a constant cosmological density parameter $\Omega_{\Lambda}=0.73$ given by the results of the WMAP experiment (Bennett et al. 2003).

We extract the initial abundances of atoms and ions out of the SBBN theoretical predictions given by Cyburt et al. (2003).

$\left[\mathrm{H}^{+}\right] \sim 0.889$

$\left[\mathrm{D}^{+}\right] \sim 2.436 \times 10^{-5}$

$\left[\mathrm{He}^{2+}\right] \sim 0.111$

$\left[\mathrm{Li}^{+}\right] \sim 3.343 \times 10^{-10}$.

Our calculations start at redshift $z_{\mathrm{i}}=10^{4}$, where the considered atoms are fully ionized, and stop at redshift $z_{\mathrm{f}}=10$ where the formation of the first structures are initiated.

In Fig. 1, we have plotted the chemistry evolution for helium, hydrogen, deuterium and lithium. Once neutral $\mathrm{He}$ is significantly abundant, see Fig. 1, charge transfer with ions is possible, allowing the formation of other neutral species, which permit the formation of $\mathrm{H}_{2}^{+}$through the exchange reaction with some neutral $\mathrm{H}$. Then, the $\mathrm{H}_{2}^{+}$charge transfer with a neutral species leads to $\mathrm{H}_{2}$ molecules. Moreover, as the radiation temperature decreases, $\mathrm{H}_{2}$ can be formed through $\mathrm{H}^{-}$by radiative attachment followed by associative detachment. Thus the two major routes of $\mathrm{H}_{2}$ formation are visible in Fig. 1 by two jumps at the redshifts $z \sim 500\left(\mathrm{H}_{2}^{+}\right.$channel) and $z \sim 150\left(\mathrm{H}^{-}\right.$channel $)$. HD formation is significant when $\mathrm{H}_{2}$ appears, the mechanism of dissociative collision between $\mathrm{H}_{2}$ and $\mathrm{D}^{+}$ions becomes efficient. $\mathrm{LiH}$ is mainly formed by exchange reaction between $\mathrm{Li}$ and $\mathrm{H}_{2}$, however the charge transfer reactions with the $\mathrm{H}^{+}$or $\mathrm{H}$ exchange reaction are important destruction mechanisms of $\mathrm{LiH}$. Thus $\mathrm{LiH}$ abundance remains low.

\section{Primordial chemistry of fluorine}

A comprehensive account was given of the chemistry of HF in interstellar clouds by Zhu et al. (2002), and more recently by Neufeld et al. (2005) for the chemistry of fluorine-bearing molecules in diffuse and dense interstellar gas clouds. One very promising result was presented by Rauscher et al. (1994) with a calculation of heavy element abundances in the context of nonhomogeneous Big Bang nucleosythesis. In this paper the relative abundance of fluorine nuclei is estimated to be close to $10^{-15}$ which is our initial abundance. Our calculations start at the redshift $z_{\mathrm{s}}=2000$ to avoid fluorine recombination. For redshifts greater than $z_{\mathrm{s}}$, the fluorine chemistry is essentially dominated by the successive recombination from $\mathrm{F}^{n+}$ to the neutral atom $\mathrm{F}$. 

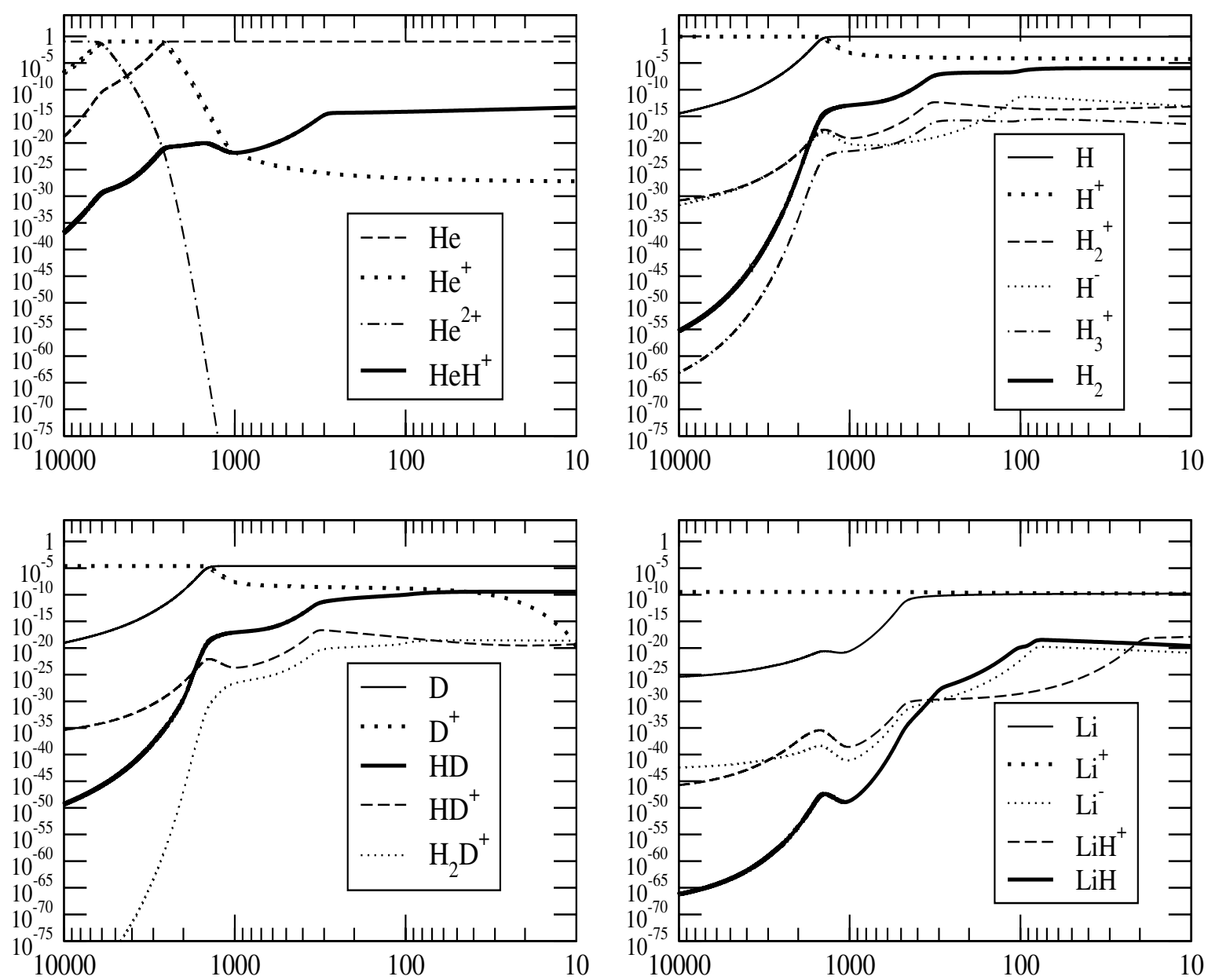

Fig. 1. Evolution of chemical abundances in the SBBC model. Vertical axes are the relative abundance and the horizontal axes are relative to the redshift. nent:

We consider seven reactions which involve fluorine compo-

- radiative association: $\mathrm{F}+\mathrm{H} \rightarrow \mathrm{HF}+\gamma$

- photodissociation: $\mathrm{HF}+\gamma \rightarrow \mathrm{F}+\mathrm{H}$;

- radiative attachment: $\mathrm{F}+\mathrm{e}^{-} \rightarrow \mathrm{F}^{-}+\gamma$

- photodetachment: $\mathrm{F}^{-}+\gamma \rightarrow \mathrm{F}+\mathrm{e}^{-}$

- associative detachment: $\mathrm{F}^{-}+\mathrm{H} \rightarrow \mathrm{HF}+\mathrm{e}^{-}$;

- electronic collision: $\mathrm{HF}+\mathrm{e}^{-} \rightarrow \mathrm{F}^{-}+\mathrm{H}$;

- neutral-neutral reaction: $\mathrm{F}+\mathrm{H}_{2} \rightarrow \mathrm{HF}+\mathrm{H}$.

In some cases we have estimated the reaction rate of these reactions. All of these reactions are coupled with the chemical network of the SBBC and the equations of evolution for the temperatures (matter and radiation) and the density.

\subsection{Radiative association}

The data on the elemental processes of formation-destruction of molecules are extremely important for the chemical kinetic calculations. In the case of the HF chemistry one of the possible channels of the HF formation is radiative association of HF in the ground electronic state. The only bound electronic state for the hydrogen halide molecules is the ground $X^{1} \Sigma^{+}$state, in which the molecules can be formed by the radiative association. There are four states correlating asymptotically with $\mathrm{F}\left({ }^{2} \mathrm{P}\right)+\mathrm{H}\left({ }^{2} \mathrm{~S}\right)$ : the lowest $\mathrm{X}^{1} \Sigma^{+}$bound state and the three lowest repulsive ${ }^{1} \Pi,{ }^{3} \Sigma^{+}$ and ${ }^{3} \Pi$ states (Brown \& Balint-Kurti 2000). The triplet states are dipole forbidden to combine radiatively with the singlet bound

state, unless spin-orbit coupling is strong which is not the case (Brown \& Balint-Kurti 2000). Therefore radiative association from $\mathrm{F}\left({ }^{2} \mathrm{P}\right)+\mathrm{H}\left({ }^{2} \mathrm{~S}\right)$ is only possible through the bound $\mathrm{X}^{1} \Sigma^{+}$and the repulsive $\mathrm{A}^{1} \Pi$ states for the HF formation:

(F1) $\mathrm{F}+\mathrm{H} \rightarrow \mathrm{HF}+\gamma$.

We can calculate the reaction rate by using the principle of detailed balance in the case where the cross section of inverse process (photodissociation of the HF) is available. Brown \& Balint-Kurti (2000) have published data on the total photodissociation cross section for $A^{1} \Pi \longleftarrow X^{1} \Sigma^{+}$which can be used to obtain the radiative association cross section:

$\sigma_{\mathrm{a}}=\frac{2 g_{\mathrm{n}}}{Z_{\mathrm{H}} Z_{\mathrm{F}}}\left(\frac{h v}{\mu c v}\right)^{2} \sigma_{\mathrm{d}}$,

Here $g_{\mathrm{n}}$ is the statistical weight, $Z_{\mathrm{H}}, Z_{\mathrm{F}}$ are statistical sums for $\mathrm{H}$ and $\mathrm{F}$ atoms respectively, $v$ is the frequency of photon, $v$ is the relative velocity of $\mathrm{H}$ and $\mathrm{F}$ atoms, $\mu$ is the reduced mass of the molecule and $\sigma_{\mathrm{d}}$ is the photodissociation cross section. Finally, the rate of the radiative association can be obtained by integration over $v$.

$k_{\mathrm{F} 1}\left(T_{\mathrm{m}}\right)=\int_{0}^{\infty} \sigma_{\mathrm{a}} f\left(T_{\mathrm{m}}, v\right) v \mathrm{~d} v$,

where $f\left(T_{\mathrm{m}}, v\right)$ is the Maxwell distribution function averaged over all angles and $T_{\mathrm{m}}$ is the matter temperature. 


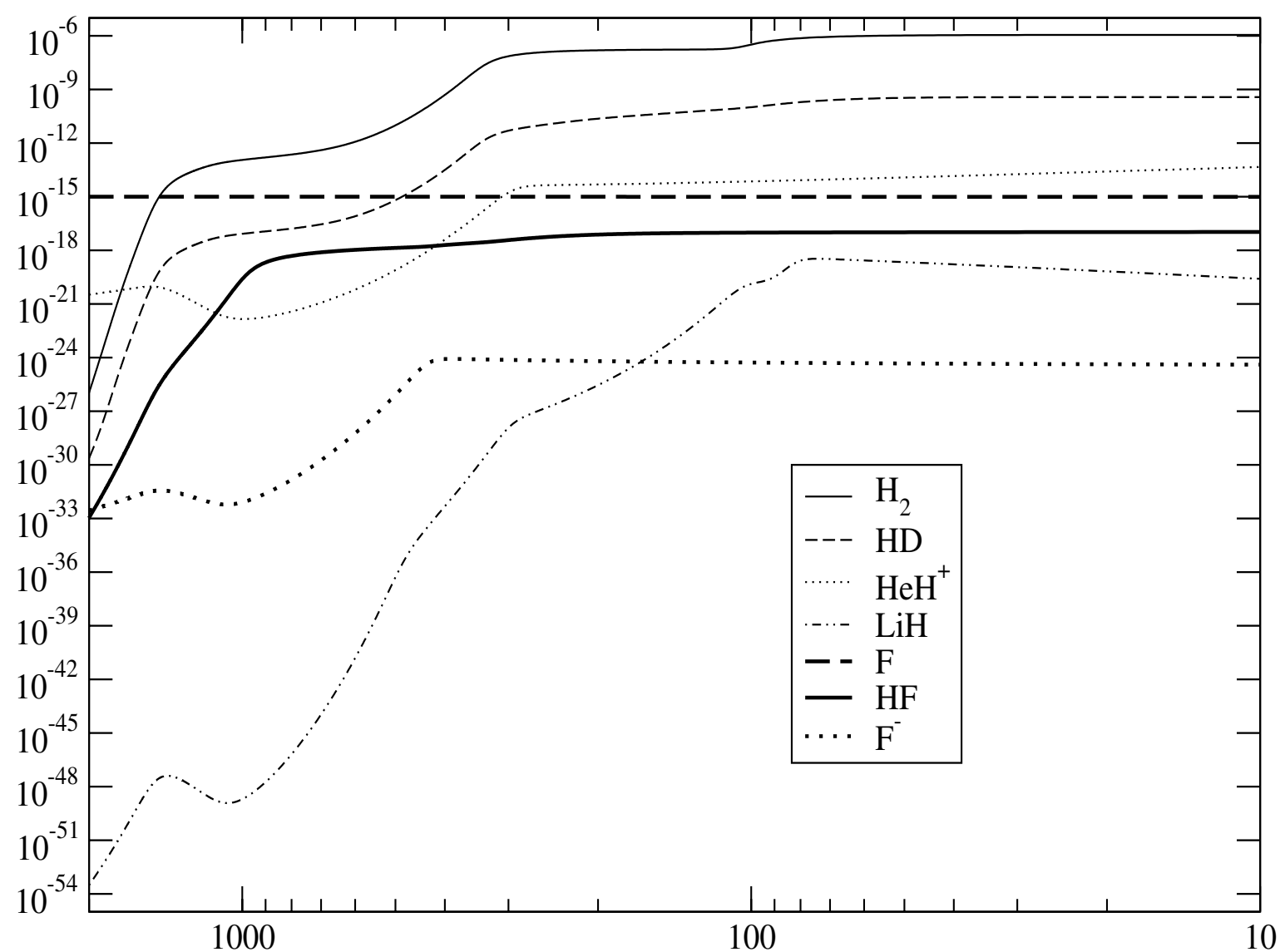

Fig. 2. Evolution of fluorine abundances compared to $\mathrm{H}_{2}, \mathrm{HD}, \mathrm{LiH}$ and $\mathrm{HeH}^{+}$abundances of $\mathrm{SBBC}$ model. Vertical axis is the relative abundance when the horizontal axis is relative to the redshift.

We find with high accuracy that the rate $k_{\mathrm{F} 1}$ can be fitted by the approximation:

$k_{\mathrm{F} 1}=1.79 \times 10^{-17} T_{\mathrm{m}}^{-0.51} \mathrm{~cm}^{3} \mathrm{~s}^{-1}$.

\subsection{Photodissociation of HF}

(F2) $\mathrm{HF}+\gamma \rightarrow \mathrm{F}+\mathrm{H}$

Using available cross sections from Brown \& Balint-Kurti (2000) for the photodissociation of HF we calculated the corresponding rate to be $k_{\mathrm{F} 2}=6.8 \times 10^{7} \exp \left(-121171 / T_{\mathrm{r}}\right) \mathrm{s}^{-1}$ where $T_{\mathrm{r}}$ is the radiation temperature.

\subsection{Radiative attachment}

(F3) $\mathrm{F}+\mathrm{e}^{-} \rightarrow \mathrm{F}^{-}+\gamma$

Considering that photodissociation of $\mathrm{F}^{-}$should be close to that of $\mathrm{Cl}^{-}$, we deduced the radiative association cross section of (F3) from the experimental cross section of $\mathrm{Cl}^{-}+\gamma \rightarrow \mathrm{Cl}+\mathrm{e}^{-}$(Rothe 1969) by using the principle of detail balance. With this cross section, we calculated the reaction rate, to be constant, and of the order of $k_{\mathrm{F} 3}=10^{-14} \mathrm{~cm}^{3} \mathrm{~s}^{-1}$.

\subsection{Photodetachment}

(F4) $\quad \mathrm{F}^{-}+\gamma \rightarrow \mathrm{F}+\mathrm{e}^{-}$

With the same hypothesis as above we used the experimental estimated cross section of the reaction $\mathrm{Cl}^{-}+$ $\gamma \rightarrow \mathrm{Cl}+\mathrm{e}^{-}$to deduce the rate constant (F4) for the photodetachment. We find an analytical expression $k_{\mathrm{F} 4}=$ $633120\left(T_{\mathrm{r}} / 300\right)^{3.6} \exp \left(-41481 / T_{\mathrm{r}}\right) \mathrm{s}^{-1}$.

\subsection{Associative detachment}

(F5) $\mathrm{F}^{-}+\mathrm{H} \rightarrow \mathrm{HF}+\mathrm{e}^{-}$.

For this reaction we have used the experimental rate constant of Fehsenfeld (Fehsenfeld et al. 1973), determined at $296 \mathrm{~K}, k_{\mathrm{F} 5}=$ $1.6 \times 10^{-9} \mathrm{~cm}^{3} \mathrm{~s}^{-1}$.

\subsection{Electronic collision}

(F6) $\mathrm{HF}+\mathrm{e}^{-} \rightarrow \mathrm{F}^{-}+\mathrm{H}$

Using the experimental cross section for this process from $\mathrm{Xu}$ et al. (2000), we calculated the rate constant to be $k_{\mathrm{F} 6}=8.1 \times$ $10^{-17} \mathrm{~s}^{-1}$ at our temperature of interest.

\subsection{Neutral-neutral reaction}

(F7) $\mathrm{F}+\mathrm{H}_{2} \rightarrow \mathrm{HF}+\mathrm{H}$. The rate coefficient has been measured, see (UMIST 1999), $k_{\mathrm{F} 7}=10^{-10} \exp \left(-400 / T_{\mathrm{m}}\right) \mathrm{cm}^{-3} \mathrm{~s}^{-1}$.

\section{Results and discussion}

Figure 2 shows clearly the formation of HF molecules. The relative abundance of fluorine species are at $z=10$ :

$-[\mathbf{F}]=9.90 \times 10^{-16}$ 
$-[\mathbf{H F}]=1.06 \times 10^{-17}$
$-\left[\mathbf{F}^{-}\right]=4.02 \times 10^{-25}$

HF molecules are mainly formed by the exothermic neutralneutral reaction of atomic fluorine with $\mathrm{H}_{2}$, which is confirmed by Snow et al. (2007) where they reported results from a survey of neutral fluorine in the interstellar medium. They showed that the hydride of fluorine, HF, is in competition with the abundance of atomic fluorine, depending on how abundant $\mathrm{H}_{2}$ is.

Primordial molecules could play an important role in the future observations of CMBR anisotropies (Dubrovich 1977; Maoli et al. 1994). These authors argued that huge enhancement of the emission line intensity from the proto-objects could occur, and mentioned that molecular protoclouds at high redshift, $10<z<300$, which have a peculiar velocity relative to the CMBR, could produce spectral-spatial fluctuations through the mecanism of pure reflection of the CMBR photons due to the opacity of a proto-object in narrow spectral lines and the Doppler shift in frequency due to the peculiar velocity. In this context, molecules of fluorine HF could play an interesting role, despite the low initial abundance of fluorine compared to the abundance of lighter elements. We find (see Fig. 2), that the abundance of molecular fluorine is the third neutral molecular abundance (with $\mathrm{H}_{2}$ and HD). Thus HF molecules could contribute to the process of fragmentation of large proto structures. HF molecules ${ }^{1}$ could be an important coolant agent in collapsing proto-objects and initiate the mechanism of thermal instability as do $\mathrm{HD}$ and $\mathrm{LiH}$ (Puy \& Signore 1996, 1997; Lipovka et al. 2005).

The detection of primordial HF will be possible in the near future with instruments such as ALMA or HERSCHEL satellite (Maoli et al. 2005). Searching for HF could result in constraints on the fundamental process of nuclei formation during the Big Bang nucleosynthesis.

Acknowledgements. We acknowledge the PNC (Programme National de Cosmologie) and the PNPS (Programme national de Physique Stellaire) for their financial assistance, as well as to Corinne Serres-Cousiné for their helpful advices. V.D. is endebted to the French CNRS for financial support through a contract of associated researcher at the University of Montpellier II.

\section{References}

Abel, T., Anninos, P., Zhang, Y., \& Norman, M. 1997, New Astron., 2, 181 Abel, T., Bryan, G., \& Norman, M. 2000, ApJ, 540, 39

Affleck, I., \& Dine, M. 1985, Nucl. Phys. B, 249, 361
Applegate, J., Hogan, C., \& Scherrer, R. 1987, Phys. Rev. D, 35, 1151 Aracil, B., Petitjean, P., Pichon, C., \& Bergeron, J. 2004, A\&A, 419, 811 Bennett, C., Halpern, M., Hinshaw, G., et al. 2003, ApJS, 148, 1

Brown, A., \& Balint-Kurti, G. 2000, J. Chem. Phys., 113, 1870

Cohen, J., Christlieb, N., Mc William, A., et al. 2004, ApJ, 612, 1167

Cyburt, R., Fields, B., \& Olive, K. 2003, Phys. Lett. B, 567, 227

Dubrovich, V. 1977, SvAL, 3, 128

Dubrovich, V. 1993, Astron. Lett., 19, 53

Dubrovich, V. 1997, A\&A, 324, 27

Fehsenfeld, F., Carleton, J., Howard, E., \& Ferguson, E. 1973, J. Chem. Phys., 58,584

Flower, D. 2000, MNRAS, 318, 875

Freedman, W. 2000, Phys. Rep., 333, 13

Galli, D., \& Palla, F. 1998, A\&A, 335, 403

Hirata, C., \& Padmanabhan, N. 2006, MNRAS, 372, 1175

Janev, R. 1974, A\&A, 37, 1

Jedamzik, K., \& Rehm, J. 2001, Phys. Rev. D, 64, 023510

Jedamzik, K., Fuller, G., Mathews, G., \& Kajino, T. 1994, ApJ, 423, 50

Kurki-Suonio, H., Jedamzik, K., \& Mathews, G. 1997, ApJ, 479, 31

Lepp, S., \& Shull, M. 1984, ApJ, 280, 465

Lepp, S., Stancil, P., \& Dalgarno, A. 2002, J. Phys. B, 35, R57

Lipovka, A., Núñez-López, R., \& Avila-Reese, V. 2005, MNRAS, 631, 850

Maoli, R., Melchiorri, F., \& Tosti, D. 1994, ApJ, 425, 372

Maoli, R., Ferrucci, V., Melchiorri, F., Signore, M., \& Tosti, D. 1996, ApJ, 457, 1

Maoli, R., Chambaud, P., Daniel, J. Y., et al. 2005, Proceedings The dusty and molecular Universe, ESA SP-577

Matsuura, S., Fujimoto, S., Nishimura, S., Hashimoto, M., \& Sato, K. 2005, Phys. Rev. D, 72, 123505

Muenter, J. 1972, J. Chem. Phys., 56, 5409

Núñez-López, R., Lipovka, A., \& Avila-Reese, V. 2006, MNRAS, 369, 2005

Neufeld, D., Wolfire M., \& Schilke, P. 2005, ApJ, 628, 260

Neufeld, D., Zmuidizinas, J., Schilke, P., \& Philipps, T. 1997, ApJ, 488, L141

Pfenniger, D., \& Puy, D. 2003, A\&A, 398, 447

Pichon, C., Scannapieco, E., Aracil, B., et al. 2003, ApJ, 597, L97

Puy, D., \& Signore, M. 1996, A\&A, 305, 371

Puy, D., \& Signore, M. 1997, New Astron., 2, 299

Puy, D., \& Pfenniger, D. 2006, A\&A, submitted

Puy, D., Alecian, G., Le Bourlot, J., Léorat, J., \& Pineau des Forêts, G. 1993, A\&A, 267, 337

Rauscher, T., Applegate, J., Cowan, J., \& Thielemann F.-K. 1994, ApJ, 429, 499

Rothe, D. E. 1969, Phys. Rev., 177, 93

Shchekinov, Y., \& Vasiliev, E. 2006, 2006 MNRAS, 368, 454

Signore, M., Vedrenne, G., De Bernardis, P., et al. 1994, ApJS, 92, 535

Songaila, A. 1996, ApJ, 561, L153

Snow, T., Destree J., \& Jensen, A. 2007, ApJ, 655, 285

Stancil, P., Lepp, S., \& Dalgarno, A. 1996, ApJ, 458, 401

Stancil, P., Lepp, S., \& Dalgarno, A. 1998, ApJ, 509, 1

Thomas, D., Schramm, D., Olive, K., et al. 1994, ApJ, 430, 291

UMIST database for astrochemistry 1999, in http//: www. udfa.net

Yoshida, N., Omukai, K., Hernquist, L., \& Abel, T. 2006, ApJ, 652, 6

Xu, Y., Gallup, G., \& Fabricant, I. 2000, Phys. Rev. A, 61, 52705

Zhu, C., Krems, R., Dalgarno, A., \& Balakrishnan, N. 2002, ApJ, 577, 795

${ }^{1}$ where the dipole moment of HF is closed to $d_{\mathrm{HF}}=1.8$ Debye, see (Muenter 1972). 